\title{
Operational approach to complementarity and duality relations
}

\author{
Alfredo Luis* \\ Departamento de Óptica, Facultad de Ciencias Físicas, Universidad Complutense, 28040 Madrid, Spain
}

(Received 12 July 2004; published 13 December 2004)

\begin{abstract}
We present a fully operational and consistent approach to complementarity. In contrast to previous approaches, in this proposal the duality relations emerge exclusively from the outcomes of simultaneous measurements performed on every run of the experiment and under the same experimental conditions. This can be done without assuming any definite relationship between the measurement performed and the complementary observables being studied.
\end{abstract}

DOI: $10.1103 /$ PhysRevA.70.062107

PACS number(s): 03.65.Ta, 03.65.Ca, 42.50.Dv, 42.50.Xa

\section{INTRODUCTION}

Complementarity means that quantum systems possess properties that are mutually exclusive: the observation of one of them precludes the observation of the other. Maybe the best illustration is provided by the interferometric waveparticle duality: if a which-path detector is arranged in order to determine the path taken by the particle within a twobeam interferometer, the interference is unavoidably disturbed.

This appealing concept without classical analog has attracted a lot of attention from the beginning of the quantum theory. However, only recently has this idea been thoroughly scrutinized. In Refs. [1-8] the interested reader can find some examples concerning formal definitions [1], quantitative evaluation [2-4], experimental observations [5-7], and investigation of its physical origin [8].

In this work we focus on the quantitative evaluations of complementarity along with their practical determination. In this regard, the most popular assessment of interferometric complementarity in the presence of a which-way detector is provided by a duality relation involving measures of the distinguishability of the path and the visibility of the interference $[2,6]$. This approach is recalled in Sec. III A.

It is worth stressing that the notion of complementarity refers to the simultaneous observations of two incompatible observables performed in every run of the experiment and under the same experimental conditions. This idea is included in every presentation of complementarity at any level. For example, in the classic interferometric wave-particle duality it is always understood that we are monitoring the visibility of the interference at the same time that we try to determine the path followed by the particle within the interferometer.

However the distinguishability of the path and the visibility of the interference developed in Refs. [2] and [6] cannot be measured simultaneously, in sharp contrast to the very spirit of complementarity discussed above. This is because the determination of the distinguishability requires not only the which-way detection whose effect is being investigated,

*Electronic address: alluis@ fis.ucm.es; URL: http://www.ucm.es/ info/gioq but also a simultaneous exact measurement of the path [see Eq. (20) below] [2,6]. This implies that the measurements of distinguishability and visibility cannot be performed simultaneously and their assessment requires different experimental arrangements. This point is confirmed by the experimental verifications of the above mentioned duality relation [6]. In this work we avoid this difficulty by addressing a genuine operational approach, where complementarity is to be measured exclusively in terms of the outputs of measurements performed simultaneously in each and every run of the experiment and under the same experimental conditions.

\section{FORMALISM}

The most general measurement is described by a positive operator measure $\Delta(w)$, where $w$ represents the outcomes appearing with probabilities $p(w)=\operatorname{tr}[\rho \Delta(w)], \rho$ being the density matrix for the system [9]. Any inference about any system property must be derived exclusively from the outcome $w$ and from the measurement performed $\Delta(w)$, which is the only information accessible to the experimenter. A very natural procedure consists in associating a quantum state $\rho_{w}$ to every outcome $w$, depending only on $w$ and $\Delta(w)$. Then we can compute the value of the desired observable in the state $\rho_{w}$.

The correspondence $\rho_{w} \leftrightarrow \Delta(w)$ can be carried out in many different ways. We just mention three natural options.

(i) As a first option we consider $\rho_{w}=|w\rangle\langle w|$ where $|w\rangle$ represents the eigenvector of $\Delta(w)$ with the largest eigenvalue. This is the best possible choice from a maximum likelihood perspective and maximizes the fidelity as shown in Ref. [10].

(ii) $\rho_{w}$ can be determined also by the whole $\Delta(w)$, instead of by part of its spectrum, in the form [11]

$$
\rho_{w}=\frac{1}{\operatorname{tr} \Delta(w)} \Delta(w) .
$$

(iii) Following a Bayesian perspective $\rho_{w}$ becomes a superposition of all possible density matrices $\rho_{\Omega}$ weighted by the probability $p(\Omega, w)=\operatorname{tr}\left[\rho_{\Omega} \Delta(w)\right]$ of the outcome $w$, 


$$
\rho_{w}=\frac{\int d \Omega p(\Omega, w) \rho_{\Omega}}{\int d \Omega^{\prime} p\left(\Omega^{\prime}, w\right)},
$$

where $\Omega$ are parameters indexing the density matrices.

In any case, the evaluation of system properties, represented by an arbitrary operator $\mathcal{M}$, can be carried out as

$$
\tilde{\mathcal{M}}=\sum_{w} p(w) \operatorname{tr}\left(\mathcal{M} \rho_{w}\right)=\operatorname{tr}\left(\mathcal{M} \rho_{D}\right),
$$

where

$$
\rho_{D}=\sum_{w} p(w) \rho_{w}
$$

represents the observed state of the system provided by the cumulative collection of the outcomes of a large number of measurements. For simplicity we have assumed that the number of measurements is large enough so that the relative frequencies of the measured outputs can be represented by the exact probabilities $p(w)$ with enough accuracy. It is also worth stressing that in principle there is no particular relation between $\Delta(w)$ and $\mathcal{M}[12]$.

This concludes the outline of the operational approach. Before considering particular examples let us stress that this procedure does not rely on the reconstruction of the input state of the system, i.e., $\rho_{D}$ is not an estimator of $\rho$. Otherwise we would be addressing the estimation of the intrinsic values of the corresponding observables, rather than the measured values that are pertinent to the notion of complementarity at work.

\section{TWO-BEAM INTERFERENCE}

We illustrate this proposal by applying it to the most relevant and widely studied example of path and visibility in two-beam interference. A brief explanation of two-beam interference is sketched in the Appendix. For two-dimensional situations the most general $\Delta(w)$ can be expressed as

$$
\Delta(w)=m_{w}+\mathbf{m}_{w} \cdot \boldsymbol{\sigma},
$$

where $\boldsymbol{\sigma}$ are the three Pauli matrices and $m_{w}, \mathbf{m}_{w}$ are real coefficients satisfying

$$
\sum_{w} m_{w}=1, \quad \sum_{w} \mathbf{m}_{w}=\mathbf{0}, \quad m_{w} \geqslant\left|\mathbf{m}_{w}\right|,
$$

in order to guarantee the reality, positivity, and normalization of $p(w)$. On the other hand, the most general density matrix and the associated statistics are

$$
\rho=\frac{1}{2}(1+\mathbf{s} \cdot \boldsymbol{\sigma}), \quad p(w)=m_{w}+\mathbf{m}_{w} \cdot \mathbf{s},
$$

where $\mathbf{s}$ is a real vector with $|s| \leqslant 1$. The parameters $\Omega$ in Eq. (2) describe a unit sphere

$$
\rho_{\Omega}=\frac{1}{2}(1+\boldsymbol{\Omega} \cdot \boldsymbol{\sigma})
$$

where

$$
\boldsymbol{\Omega}=\left(\begin{array}{c}
r \sin \theta \cos \phi \\
r \sin \theta \sin \phi \\
r \cos \theta
\end{array}\right), \quad d \Omega=r^{2} \sin \theta d r d \theta d \phi .
$$

The three correspondences $\rho_{w} \leftrightarrow \Delta(w)$ discussed above can be summarized by the single expression

$$
\rho_{w}=\frac{1}{2}\left(1+\frac{1}{\eta_{w}} \mathbf{m}_{w} \cdot \boldsymbol{\sigma}\right),
$$

where for (i) $\eta_{w}=\left|\mathbf{m}_{w}\right|$, for (ii) $\eta_{w}=m_{w}$, while for (iii) $\eta_{w}$ $=5 m_{w}$. Thus the observed state is

$$
\rho_{D}=\frac{1}{2}(1+\boldsymbol{\mu} \cdot \boldsymbol{\sigma}), \quad \boldsymbol{\mu}=\sum_{w} \frac{1}{\eta_{w}}\left(m_{w}+\mathbf{m}_{w} \cdot \mathbf{s}\right) \mathbf{m}_{w} .
$$

The path variable is represented by the operator $\sigma_{z}$, while the phase observable is given by the positive operator measure $|\phi\rangle\langle\phi|$ where $|\phi\rangle$ are the phase states [4,13,14],

$$
|\phi\rangle=\frac{1}{\sqrt{2 \pi}}\left(|-\rangle+e^{i \phi}|+\rangle\right),
$$

$| \pm\rangle$ are the eigenstates of $\sigma_{z}$, and $\phi$ can take any value in a $2 \pi$ interval. A suitable operator representing the phase is the complex exponential of $\phi$,

$$
E=\int_{2 \pi} d \phi e^{i \phi}|\phi\rangle\langle\phi|=|-\rangle\langle+| .
$$

Usually the measurement of $\sigma_{z}$ corresponds to a measurement of photon number, atomic populations, or spin orientation. The procedure to measure the phase $\phi$ is sketched in the Appendix.

A suitable measure of fluctuations of observables in finitedimensional Hilbert spaces is given by the certainties

$$
\mathcal{C}_{z}=\left|\left\langle\sigma_{z}\right\rangle\right|, \quad \mathcal{C}_{\phi}=|\langle E\rangle|
$$

representing the degree of certainty one can have concerning the value of the corresponding observable [4]. It must be noted that $\mathcal{C}_{z}, 2 \mathcal{C}_{\phi}$ coincide with the standard definitions of predictability and visibility, respectively $[2,4]$. They are bounded by $1 \geqslant \mathcal{C}_{z} \geqslant 0, \frac{1}{2} \geqslant \mathcal{C}_{\phi} \geqslant 0$, and satisfy the duality relations [4]

$$
\mathcal{C}_{z}^{2}+4 \mathcal{C}_{\phi}^{2} \leqslant 1, \quad \mathcal{C}_{z} \mathcal{C}_{\phi} \leqslant \frac{1}{4}
$$

\section{A. Standard approach}

In order to illustrate the properties of the formalism introduced in this work we recall in the first place the results of applying the standard approach to the case of two-beam interference $[2,6]$.

The which-way detection is obtained by coupling the system to auxiliary degrees of freedom, i.e., an apparatus, initially in a given state $|M\rangle$. In practical terms this can be the internal degrees of freedom of the interfering particle, such as the internal electronic state of an atom or the polarization state of a photon. The standard system-apparatus coupling is of the form 


$$
U=V_{+}|+\rangle\left\langle+\left|+V_{-}\right|-\right\rangle\langle-|,
$$

leading to an output state

$$
\widetilde{\rho}=U \rho \otimes|M\rangle\langle M| U^{\dagger},
$$

where $V_{ \pm}$are unitary operators acting solely on the apparatus, $\left|M_{ \pm}\right\rangle=V_{ \pm}|M\rangle$. Thus, the state of the apparatus experiences different transformations depending on the path. The path can then be disclosed with minimum error by performing a measurement on the apparatus described by projection on the orthogonal vectors $\left|\tilde{M}_{ \pm}\right\rangle[4]$ :

$$
\left|\tilde{M}_{ \pm}\right\rangle=\frac{\cos (\theta / 2)}{\cos \theta}\left|M_{ \pm}\right\rangle-\frac{\sin (\theta / 2)}{\cos \theta}\left|M_{\mp}\right\rangle,
$$

where $\sin \theta=\left\langle M_{-} \mid M_{+}\right\rangle$, assumed to be real without loss of generality. The reduced states of the system after the whichway detection are $\rho_{j}=\left\langle\tilde{M}_{j}|\widetilde{\rho}| \tilde{M}_{j}\right\rangle / p_{j}, j= \pm 1$, where

$$
\begin{aligned}
\left\langle\tilde{M}_{j}|\widetilde{\rho}| \tilde{M}_{j}\right\rangle= & \frac{1}{4}\left[1+j s_{z} \cos \theta+\left(s_{z}+j \cos \theta\right) \sigma_{z}+\sin \theta\left(s_{x} \sigma_{x}\right.\right. \\
& \left.\left.+s_{y} \sigma_{y}\right)\right],
\end{aligned}
$$

and $p_{j}=\left(1+j s_{z} \cos \theta\right) / 2$.

For the approach developed in Refs. [2] and [6] the predictability and visibility (i.e., the certainties $\mathcal{C}_{z}^{\prime}, \mathcal{C}_{\phi}^{\prime}$ ) are given by the average of the predictabilities and visibilities associated to each $\rho_{j}[2,6]$ :

$$
\begin{gathered}
\mathcal{C}_{z}^{\prime}=\sum_{j} p_{j}\left|\left\langle+\left|\rho_{j}\right|+\right\rangle-\left\langle-\left|\rho_{j}\right|-\right\rangle\right|=\sum_{j} \mid\left\langle\tilde{M}_{j}|\langle+|\widetilde{\rho}|+\rangle| \tilde{M}_{j}\right\rangle \\
-\left\langle\tilde{M}_{j}|\langle-|\tilde{\rho}|-\rangle| \tilde{M}_{j}\right\rangle \mid=\max \left(\mathcal{C}_{z},|\cos \theta|\right) \\
\mathcal{C}_{\phi}^{\prime}=\sum_{j} p_{j}\left|\left\langle+\left|\rho_{j}\right|-\right\rangle\right|=|\sin \theta| \mathcal{C}_{\phi}
\end{gathered}
$$

where $\mathcal{C}_{z}, \mathcal{C}_{\phi}$ are the intrinsic certainties computed using $\rho$.

Note that in order to determine $\mathcal{C}_{z}^{\prime}$ experimentally it is necessary to perform simultaneously a measurement on the apparatus (projection on $\left|\tilde{M}_{j}\right\rangle$ ) and an exact measurement on the system (projection on $| \pm\rangle$ ). Therefore there is no room for the measurement of the visibility on the same elements of the ensemble. It must then be determined by measurements performed on other elements of the ensemble observed under different experimental conditions.

\section{B. Operational approach}

Next we apply the operational approach to the above interferometric arrangement with path detection. The main difference from the above analysis is that for the approach presented in this work the joint measurement of $\left|\tilde{M}_{ \pm}\right\rangle$on the apparatus and phase $|\phi\rangle$ on the system after the coupling (16) is enough to obtain quantitative duality relations. Note that in this case the same measurement is performed on each and every element of the ensemble and always under the same experimental conditions.

The statistics of the simultaneous measurement of path and phase can be expressed as

$$
\operatorname{tr}\left(\tilde{\rho}|\phi\rangle\left\langle\phi|\otimes| \tilde{M}_{j}\right\rangle\left\langle\tilde{M}_{j}\right|\right)=\operatorname{tr}[\rho \Delta(j, \phi)],
$$

where $\Delta(w)$ with $w=(j, \phi)$ is the positive operator measure

$$
\Delta(j, \phi)=\frac{1}{4 \pi}\left[1+j \cos \theta \sigma_{z}+\sin \theta\left(\cos \phi \sigma_{x}-\sin \phi \sigma_{y}\right)\right],
$$

$j= \pm 1, \phi$ takes any value in a $2 \pi$ interval, and the parameter $\theta$ expresses the relative accuracy of the phase and path observations [15]. For $\theta=0$ we have an exact measurement of the path while the phase is fully uncertain, and vice versa for $\theta=\pi / 2$.

In the most general case the observed state is

$$
\rho_{D}=\frac{1}{2}\left[1+\nu s_{z} \cos ^{2} \theta \sigma_{z}+\frac{\nu}{2} \sin ^{2} \theta\left(s_{x} \sigma_{x}+s_{y} \sigma_{y}\right)\right],
$$

where $\nu=1$ for options (i) and (ii), while $\nu=1 / 5$ for option (iii).

In this case the observed certainties are simply related to the intrinsic ones in the form

$$
\tilde{\mathcal{C}}_{z}=\nu \cos ^{2} \theta \mathcal{C}_{z}, \quad \tilde{\mathcal{C}}_{\phi}=\frac{\nu}{2} \sin ^{2} \theta \mathcal{C}_{\phi},
$$

where the variables with tildes refer to the observed values (computed using $\rho_{D}$ ) while the certainties without tildes refer to the intrinsic values (computed using $\rho$ ). Using Eq. (15) for the intrinsic values we get the duality relations for the observed certainties,

$$
\begin{aligned}
& \widetilde{\mathcal{C}}_{z}{ }^{2}+4 \widetilde{\mathcal{C}}_{\phi}^{2} \leqslant \nu^{2} \max \left\{\cos ^{4} \theta, \frac{1}{4} \sin ^{4} \theta\right\}, \\
& \widetilde{\mathcal{C}}_{z} \widetilde{\mathcal{C}}_{\phi} \leqslant \frac{\nu^{2}}{8} \sin ^{2}(2 \theta) \mathcal{C}_{z} \mathcal{C}_{\phi} \leqslant \frac{\nu^{2}}{32} \sin ^{2}(2 \theta) .
\end{aligned}
$$

These expressions (24) and (25) properly reflect in a fully operational way the notion of complementarity at work including the characteristics of the measuring process.

For this particular example of two-beam interferometry some other operational duality relations have been examined before being computed directly in terms of the outputs of a noisy joint measurement of the corresponding observables $[3,4]$. This sort of approach focuses on the outcomes $w$ of the measurement, disregarding most of the information contained in $\Delta(w)$. Moreover, it requires prior assumptions concerning the relation between the outputs of the measurement and the values of the system variables.

On the other hand, the procedure presented here is entirely different since it does not rely on any prior relation between the measurement and the observables being investigated. This lack of prior assumptions is relevant since the relation between the measurement outputs and the observables being studied is necessarily perturbed by the unavoidable unsharpness of the joint measurement of complementary observables. Moreover, the inferences about system properties in this proposal naturally embody the characteristics of the measurement being performed through the dependence of $\rho_{w}$ and the measured state $\rho_{D}$ on $\Delta(w)$. Therefore, the for- 


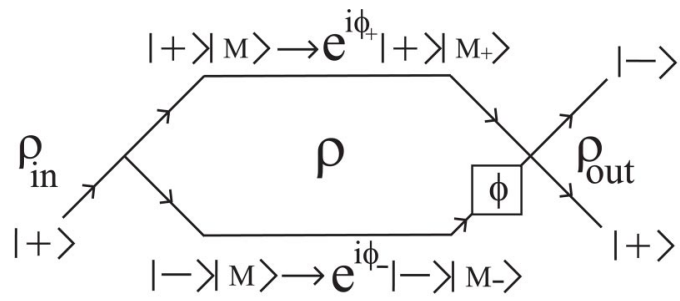

FIG. 1. Scheme of a two-beam interferometer illustrating path detection and phase-difference measurement.

malism addressed here makes a more sound use of the information provided by the measurement.

\section{CONCLUSIONS}

We have presented a fully operational and consistent approach to complementarity. In contrast to previous formalisms, in this proposal quantitative duality relations emerge exclusively from the outcomes of simultaneous measurements of complementary variables performed always under the same experimental conditions. The only ingredients used are the outputs of the measurement and our knowledge of the measurement being performed. It is not necessary to assume any definite relation between the measured outputs and the system variables under investigation. This is interesting, given the natural unsharpness of any attempt to a joint measurement of incompatible observables. We think that this approach offers a perspective on complementarity especially suitable for experimental observations of this phenomenon.

\section{APPENDIX: TWO-BEAM INTERFERENCE}

In this appendix we provide a basic illustration of twobeam interference schematized in Fig. 1. This is actually the most common and simple implementation of the idea of interference as the superposition of two waves. These waves are the two internal paths within the interferometer. This framework includes classic interferometers such as the double slit, the Michelson and Mach-Zehnder interferometers, as well as more sophisticated realizations [7].

Focusing on a quantum description, the two paths can be formally described by two orthonormal states $| \pm\rangle$, so that the state of the system $\rho$ within the interferometer is in general a superposition of these two path states $| \pm\rangle$. These states $| \pm\rangle$ can be photon-number states (such as $|+\rangle=|1,0\rangle,|-\rangle=|0,1\rangle$, where $\left|n_{+}, n_{-}\right\rangle$represent $n_{+}$photons in the upper path and $n_{-}$ photons in the lower path), atomic internal energy levels (as in Ramsey interferometry), or the orientation of a component of a $1 / 2$ spin.

The first element of a two-beam interferometer (the input beam splitter) is devised to produce a coherent superposition of $| \pm\rangle$ from an initial input state $\rho_{\text {in }}$, such as $\rho_{\text {in }}=|+\rangle\langle+|$ for example, via a transformation of the form $| \pm\rangle \rightarrow(|+\rangle \pm \mid$ $-\rangle) / \sqrt{2}$.

Then the vectors $| \pm\rangle$ experience different evolutions leading to the appearance of a phase difference $\phi_{+}-\phi_{-}$between the two paths. The purpose of any interferometric setup is the detection or monitoring of the phase difference via a measurement performed on the output state $\rho_{\text {out }}$.

In our case the phase difference is represented in the quantum domain by the states $|\phi\rangle$ [14]. It can be appreciated that $\phi$ in Eq. (12) is the relative phase between the two paths $| \pm\rangle$. Therefore, the measurement of the phase difference corresponds to the projection of the internal state $\rho$ of the interfering system on the states $|\phi\rangle$.

To this end the output state is obtained after adding a phase shift $\phi$ to one of the paths and then mixing the two internal paths at an output beam splitter (or beam merger) producing the transformation $| \pm\rangle \rightarrow(|+\rangle \pm|-\rangle) / \sqrt{2}$. These two steps (phase shift and beam splitting) lead to the following transformations between internal and output states:

$$
|\phi\rangle \rightarrow|+\rangle, \quad|\phi+\pi\rangle \rightarrow|-\rangle .
$$

With this we get that the detection of the states $| \pm\rangle$ at the output corresponds to the projection of the internal state $\rho$ on the phase-difference states $|\phi\rangle$.

On the other hand, the standard procedure of path detection involves the use of additional degrees of freedom (initially in a given state $|M\rangle$ ) that experience different transformations depending on the path $| \pm\rangle|M\rangle \rightarrow| \pm\rangle\left|M_{ \pm}\right\rangle$. A very convenient choice for these auxiliary degrees of freedom are the internal state of the interfering particle (the internal electronic state of an atom or the polarization of a photon). Finally, a measurement is performed on the output state of the auxiliary variables. Since the initial state of the auxiliary variables is in a fixed state known in advance, the information obtained in this measurement can be regarded as information exclusively about the path followed.
[1] M. O. Scully, B.-G. Englert, and H. Walther, Nature (London) 351, 111 (1991); S. Dürr and G. Rempe, Am. J. Phys. 68, 1021 (2000); P. Busch et al., J. Phys. A 34, 5923 (2001); M. Mei and M. Weitz, Phys. Rev. Lett. 86, 559 (2001); A. Luis, J. Phys. A 34, 8597 (2001); Phys. Rev. Lett. 88, 230401 (2002).

[2] B.-G. Englert, Phys. Rev. Lett. 77, 2154 (1996); G. Björk and A. Karlsson, Phys. Rev. A 58, 3477 (1998); B.-G. Englert and J. A. Bergou, Opt. Commun. 179, 337 (2000).
[3] G. Björk et al., Phys. Rev. A 60, 1874 (1999); O. Steuernagel, e-print quant-ph/9908011.

[4] A. Luis, Phys. Rev. A 64, 012103 (2001); 67, 032108 (2003).

[5] X. Y. Zou, L. J. Wang, and L. Mandel, Phys. Rev. Lett. 67, 318 (1991); E. Buks et al., Nature (London) 391, 871 (1998); S. Dürr, T. Nonn, and G. Rempe, ibid. 395, 33 (1998); P. Bertet et al., ibid. 411, 166 (2001).

[6] S. Dürr, T. Nonn, and G. Rempe, Phys. Rev. Lett. 81, 5705 
(1998); P. D. D. Schwindt, P. G. Kwiat, and B.-G. Englert, Phys. Rev. A 60, 4285 (1999); S. Dürr and G. Rempe, Opt. Commun. 179, 323 (2000).

[7] M. Brune et al., Phys. Rev. A 45, 5193 (1992); L. Davidovich et al., Phys. Rev. Lett. 71, 2360 (1993); M. Brune et al., ibid. 77, 4887 (1996); L. Davidovich et al., Phys. Rev. A 53, 1295 (1996); J. M. Raimond, M. Brune, and S. Haroche, Phys. Rev. Lett. 79, 1964 (1997); S. Haroche, Phys. Today 51 (7), 36 (1998).

[8] S. M. Tan and D. F. Walls, Phys. Rev. A 47, 4663 (1993); P. Storey et al., Nature (London) 367, 626 (1994); 375, 368 (1995); B.-G. Englert, M. O. Scully, and H. Walther, ibid. 375, 367 (1995); H. Wiseman and F. Harrison, ibid. 377, 584 (1995); L. S. Schulman, Phys. Lett. A 211, 75 (1996); H. M. Wiseman et al., Phys. Rev. A 56, 55 (1997); P. Knight, Nature (London) 395, 12 (1998); A. Luis and L. Sánchez-Soto, Phys. Rev. Lett. 81, 4031 (1998); J. Opt. B: Quantum Semiclassical Opt. 1, 668 (1999).

[9] C. W. Helstrom, Quantum Detection and Estimation Theory
(Academic Press, New York, 1976); A. Peres, Found. Phys. 20, 1441 (1990); Quantum Theory: Concepts and Methods (Kluwer Academic, Dordrecht, 1993); W. M. de Muynck, Foundations of Quantum Mechanics, An Empiricist Approach (Kluwer Academic, Dordrecht, 2002).

[10] J. Audretsch, L. Diósi, and Th. Konrad, Phys. Rev. A 68, 034302 (2003); e-print quant-ph/0210205.

[11] H. F. Hofmann, Phys. Rev. A 67, 022106 (2003).

[12] M. J. W. Hall, Phys. Rev. A 69, 052113 (2004).

[13] T. S. Santhanam, Phys. Lett. 56A, 345 (1976); S. M. Barnett and D. T. Pegg, J. Mod. Opt. 36, 7 (1989); D. Ellinas, J. Math. Phys. 32, 135 (1991); M. Grabowski, Int. J. Theor. Phys. 28, 1215 (1989); Rep. Math. Phys. 29, 377 (1991).

[14] A. Luis and L. L. Sánchez-Soto, Phys. Rev. A 48, 4702 (1993); Progress in Optics, edited by E. Wolf (Elsevier, Amsterdam, 2000), Vol. 41, p. 421.

[15] W. M. de Muynck and H. Martens, Phys. Rev. A 42, 5079 (1990); P. Busch and C. R. Shilladay, ibid. 68, 034102 (2003); e-print quant-ph/0207081. 